\title{
Nucleus ventralis oralis anterior a potential target in deep brain stimulation for post anoxic dystonia?
}

\begin{abstract}
Deep brain stimulation (DBS) effectiveness in secondary dystonia is still debated. Different targets have been utilized, thalamic (Vim), zona incerta, sub thalamic nucleus (STN), and globus pallidus internus (GPi) with generally disappointing clinical results. Structural lesions of the GPi, the target of choice in Dystonia, are found in some cases of secondary dystonia and prevent us from treating these patients with DBS implantation. We report the third published case of a patient with severe generalized post anoxic dystonia, associated with bilateral necrosis of the GPi, who showed significant improvement after DBS procedure in the thalamic Ventralis oralis anterior (Voa). The Ventral oralis anterior nucleus; pallidal receiving area in the thalamic nuclei could be an alternative target.
\end{abstract}

Keywords: post-anoxic dystonia, deep brain stimulation, thalamus, ventral oralis anterior, ventral lateral nucleus, secondary dystonia
Volume 9 Issue 4 - 2019

\author{
Maher Mansour, ${ }^{Y}$ Yasser-M Mansour, ${ }^{2}$ Leila \\ Mansour, Janardan Vaidyanathan ${ }^{3}$ \\ 'Department of Neurosurgery, Mediclinic AI Noor Hospital, \\ United Arab Emirates \\ ${ }^{2}$ University of Western Sydney, Australia \\ Medtronic MEA, India
}

\begin{abstract}
Correspondence: Maher Mansour, Department of
Neurosurgery, Mediclinic Al Noor Hospital, P.O.Box 467I 3, Abu

Dhabi, United Arab Emirates, Tel 00971504456722,
\end{abstract}

Email Mansour maher@hotmail.com

Received: July 20, 2019 | Published: July 30, 2019
Abbreviations: DBS, deep brain stimulation; STN, sub thalamic nucleus; GPi, globus pallidus internus; Voa, ventrolateral thalamic nuclei; MRI, ventrolateral thalamic nuclei

\section{Introduction}

Deep brain stimulation (DBS) of the thalamus (Vim), zona incerta, sub thalamic nucleus (STN), and globus pallidus internus (GPi) as well as pallidotomy (GPi) and thalamotomy (ventro-intermediomedialis [Vim], ventro-oralis posterior [Vop]) have been reported to improve generalized or focal dystonia. ${ }^{1-15}$ Thalamic DBS for dystonia may increase the dystonic symptoms, especially when the Vim nucleus is the target of the stimulation. ${ }^{16}$ Targeting the thalamus was abandoned because several publications suggested that the Globus pallidum internus ( $\mathrm{GPi}$ ) is a better choice for stereotactic treatment in patients with dystonia. ${ }^{14,17-21}$ Structural lesions of the GPi might be an obstacle for the DBS electrode placement and stimulation; therefore, the Ventralis oralis anterior (Voa) nucleus (Ventral lateral anterior in the Anglo-American terminology), pallidal receiving area in the thalamic nuclei, could be an alternative target. ${ }^{22-25}$

There has been only two reports of the efficacy of DBS in post anoxic, dystonia with necrosis of the pallida, although this is one of the most frequent causes of generalized dystonia. ${ }^{23,24}$ We report the case of a patient with severe generalized post anoxic dystonia, and bilateral structural abnormalities of the GPi, who showed dramatic improvement 18 months after thalamic Ventralis oralis anterior nucleus DBS.

\section{Material and methods}

\section{Patient history}

The patient was a 26year-old male, drug-abuser, and experienced a 3-day hypoxic coma, in unknown circumstances. During the following months, he gradually developed generalized dystonic movements with spastic paraparesis, which evolved during the next years to generalized dystonia, progressively worsening and confining at best the patient to the use of a wheelchair, but mostly bedridden. When he was evaluated at our institution, 13years following the accident; he had generalized dystonia in all four limbs mainly in extension, predominant torsional dystonia in neck with left laterocollis and retrocollis and opisthotonic position of the trunk. He had spasticity and dystonic upper limbs, bilateral wrist and fingers deformity (Figure 1).

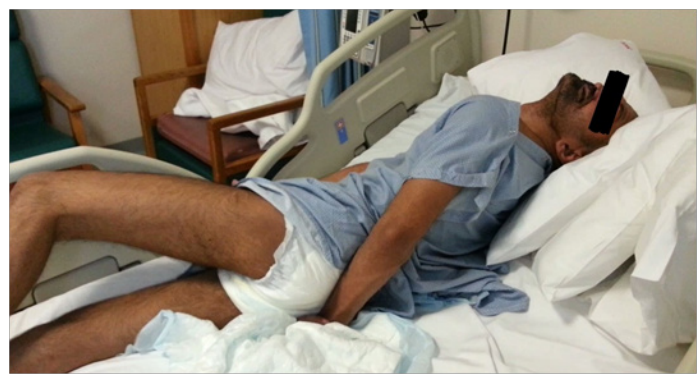

Figure I Pre-operative condition showing opisthotonic position in the bed, with retro and laterocollis.

He was aphonic, communicating with his entourage with his right thumb and index finger only, but had apparent orientation to time, place and persons with coherent answers. Severe pain was present in the neck, left shoulder, wrists and sometimes in the ankles. He was unable to walk or stand for 10years, aphonic for 5years, fed through PEG tube since 3years and was totally dependent in all daily living activities.

Burke-Fahn-Marsden rating scale ${ }^{26}$ (BFMRS) score was: 104/120. A T2 weighted brain Magnetic Resonance imaging (MRI) scan showed hypersignal bilaterally involving both pallida, mostly affecting the most medial part (Figure 2). Treatment attempts with Trihexyphenidyl, Carbidopa-Levodopa, Carbamazepine, Clonazepam, Phenytoin and Baclofen were unsuccessful. Botulinum toxin injections provided some relief of the muscular contractions but rapidly became ineffective considering the importance of dystonic movements. The family gave informed consent for DBS surgery. As the main output of the pallidum is the ventrolateral thalamic nuclei (Voa) and the centromedian-parafascicular nucleus, ${ }^{30,34}$ we decided to target Voa for DBS and hoped it would act directly on the last distal part of the retroactive feedback to the motor cortex from GPi. 


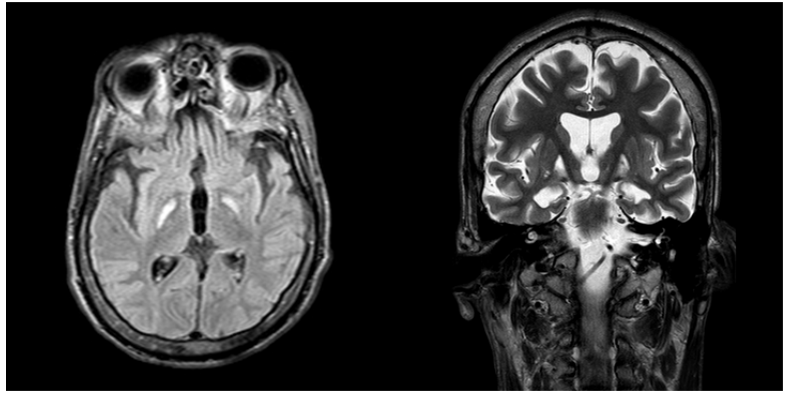

Figure 2 Pre-operative MRI showing selective necrosis of the Globus Pallidus interna, predominantly on the right side (above images are in radiological view).

\section{Radiological exams}

Stereotactic MRI was used to plan the target and trajectory (Siemens, 1.5 Tesla). All the sequences taken pre and post operatively confirmed to standard neuronavigation protocols- no gap, no interleaving, no angulations, square matrix and contiguous slices which were $1-2 \mathrm{~mm}$ thick. 3D T1 sequences were acquired with an isotropic voxel $(1 \mathrm{~mm})$ from the hard pallet to the vertex without contrast and with double dose contrast. 30 slices of $2 \mathrm{~mm}$ thick T2 coronals were acquired; this sequence was centered at mid-commissural point (MCP). The length of AC-PC and width of third ventricle was measured at MCP. Height of the thalamus was measured on $\mathrm{T} 2$ coronals.

\section{Surgical technique}

Under general anesthesia, the patient underwent stereotactic implantation of quadripolar electrodes (lead model 3387, Medtronic, Minneapolis, MN) in the Voa bilaterally, using MRI-guided stereotactic surgery (Leksell-G frame, Elekta, Stockholm, Sweden) and macro electrode stimulation (using the Leksell Neuro-Generator, Elekta, Stockholm, Sweden) to rule out side effects caused by proximity to the posterior limb of the internal capsule under controlled general anesthesia.

Coordinates for Voa were, according to Guiot scheme, ${ }^{27} 0.5 \mathrm{~mm}$ posterior to the mid-commissure point (MCP) in order to reach the posterior part of Voa and anterior part of Vop, $10 \mathrm{~mm}$ lateral and at the level of the anterior commissure-posterior commissure (AC-PC) plane (Figure 3). Only indirect visualization was possible as conventional MRI sequences cannot delineate different thalamic nuclei.

The planned electrode position was at the base of the Voa nucleus close to the Voa/Vop border (Figure 4). After carefully incising the dura at the marked point of entry a monopolar lesion making electrode (model 1017043 from Elekta, Stockholm, Sweden) was used to create a track for passage of the permanent lead. Impedance was monitored continuously to differentiate white or grey matter. Once the target was reached, macro stimulation was performed using the Leksell Neuro Generator up to supramaximal threshold of amplitude. Intraoperatively lateral fluoroscopy was used to verify the lead path and location. The actual position of the electrode was evaluated postoperatively by CT scan in stereotactic conditions and audited in the Leksell Surgiplan software (Version 10.1, Elekta, Stockholm, Sweden) (Figure 5). The difference between the planned and actual stereotactic target coordinates was up to a maximum of $0.5 \mathrm{~mm}$ in lateral, anteriorposterior and vertical axis. The implantable neurostimulator (INS) Activa PC was implanted thereafter.

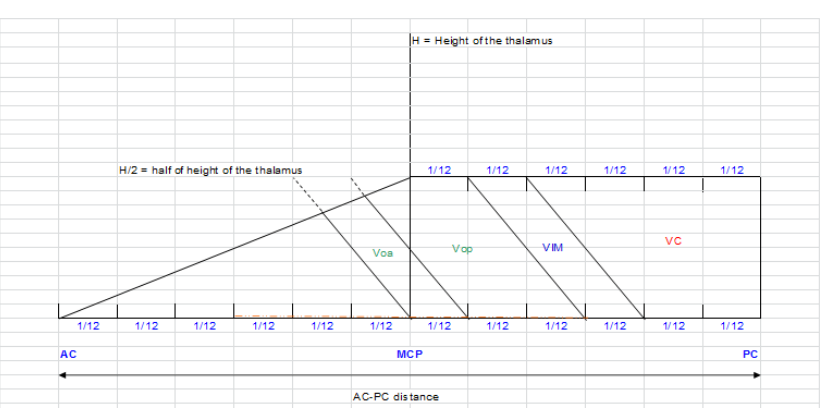

Figure 3 Guiot Scheme, 10mm from midline AC-PC showing the Voa, Vop and Vim.
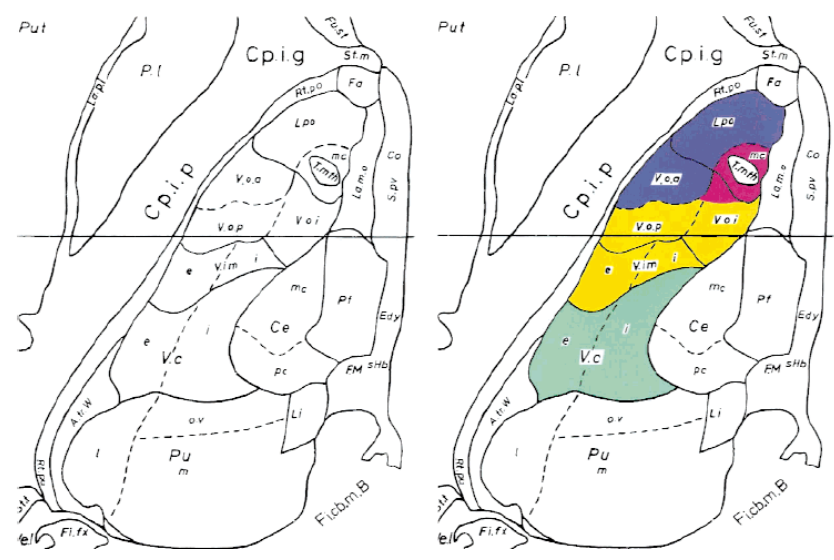

Figure 4

Left The horizontal plate from the atlas of Schaltenbrand \& Wahren ${ }^{28}$ with Hassler's ${ }^{29}$ nuclear outlines at $2 \mathrm{~mm}$ above AC-PC plane, showing the position of the planned electrode.

Right: Same plate as at left with superimposed color-coded subcortical afferent territories extrapolated from comparable horizontal level of the monkey 24 thalamus from the atlas by llinsky et al. ${ }^{30}$ Somatosensory territory is shown in green, cerebellar territory in yellow, pallidal territory in navy blue, and nigral territory in burgundy. All abbreviations are as in the atlas by Schaltenbrand and Wahren.
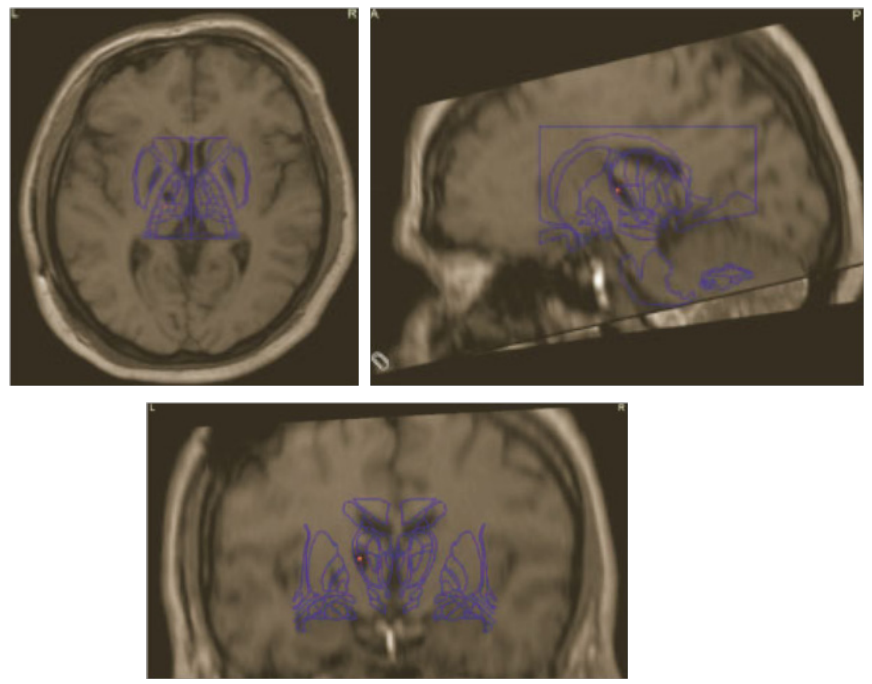

Figure $\mathbf{5}$ The correct position of the electrode was evaluated postoperatively by CT scan in stereotactic conditions and the Surgiplan software. 
Initial programming was performed on the following day as no microlesion effect was observed. The initial postoperative stimulation parameters were 2.5 volts, $185 \mathrm{~Hz}, 250$ microseconds, with monopolar stimulation on contacts 1 and 2 on the left Voa, 9 and 10 on the right side. Further adjustments of the stimulation parameters were performed during the follow-up period. Chronic stimulation was in the continuous mode. The amplitude was augmented bilaterally to 3.5 volts.

\section{Results}

Follow-up was performed at weekly intervals using the BFM rating scale. This scale is the sum of itemized scores obtained by multiplying the severity score by provoking factor by weight factor for each sub item for eyes, mouth, speech and swallowing, neck, arm and leg, and trunk, with a total maximal score of 120(26). Formal cognitive testing was not possible in this patient as he was unable to verbally communicate. During the first 2 weeks, no significant changes were observed in his condition as compared with his pre-operative status.

After the third week, the patient was able to stand unaided and could walk with assistance, with only moderate persistent left proximal dystonia, retrocollis, laterocollis, and hesitation on changing directions (Figure 6). The BFM dystonia scale improved by $48 \%$ in total $(104 / 120$ pre-op, $54 / 120$ post-op); disability scale improved from $13 / 30$ to $6 / 30$ and movement scale improved from $20 / 120$ to $2 / 120$. The functional status and the daily life activities were gradually improved after 18 months postoperatively.

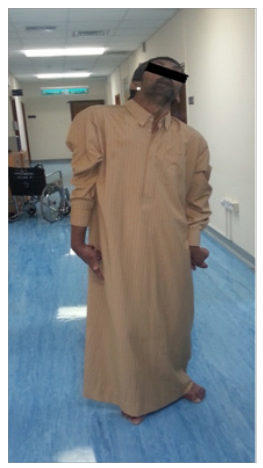

Figure $\mathbf{6}$ The patient is now able to walk again with the stimulator always on. He still suffers intermittently, under stress conditions, from jerky movements of the left hand, but the movements of the right upper limb are less prominent compared with the preoperative condition.

\section{Discussion}

Deep brain stimulation is now worldwide approved for the treatment of refractory Parkinson's disease, essential tremor and dystonia. It has been considered for new applications in a variety of other conditions including chronic pain, epilepsy and psychiatric disorders. ${ }^{1,2,9,11,17,32}$ The mechanism of action remains debated and the scope of clinical applications continue to fascinate the specialized physicians.

The globus pallidus internus was the first targeted structure for deep brain stimulation, to show significant improvement in patients with generalized dystonia without basal ganglia lesions. ${ }^{1,17,20,21,33}$ Usually the time delay, before onset of improvement, is about 3 months in observations with thalamotomy, Pallidotomy., ${ }^{4,510,12}$ and bilateral GPi-DBS. ${ }^{8,17}$

Structural brain lesions are common in secondary dystonia. ${ }^{31}$ It is possible that lesions of the GPi may result in loss of inhibitory pallidal projections to the thalamus and thus, provoke the hyperkinetic condition. ${ }^{23}$ In postanoxic dystonia, lesions in the GPi may impair the effect of GPi-DBS.

The main stimuli of the pallidum are through the Ventral oralis anterior nucleus (Ventral lateral anterior in the Anglo-American terminology) and the centromedian-parafascicular nucleus. ${ }^{23}$ Targeting the Voa for DBS could act directly on the last distal link in the retroactive feedback system to the motor cortex of the GPi. ${ }^{23,30,34}$ Only 3 weeks after Voa-DBS, Our patient with generalized post anoxic dystonia had dramatically improved with important regression of dystonic movements, possible assisted gait, and partial autonomy in this patient who previously had used a wheelchair and was bedridden for more than 10years. The improvement has involved all areas of the body.

Side effects of the DBS have been difficult to estimate because of the difficulties to communicate with this patient.

To our knowledge, there are only 2 reported cases of post anoxic dystonia treated successfully with Voa DBS. The first case report was published in 2002 by Ghika et al. ${ }^{24}$ They have reported one patient with generalized post anoxic dystonia was first treated with DBS of GPi without significant improvement then with DBS of Voa. The patient showed a dramatic improvement after the second DBS procedure. The placement of the electrode in the Voa nucleus was confirmed by autopsy, as the patient committed suicide 4 months after the procedure.

The second case, in 2009 published by Constantoyannis, ${ }^{23}$ the position of the electrodes in the Voa nucleus was confirmed by a postoperative MRI. The follow-up, over a period of 28months, revealed that the Voa nucleus offers, not only temporary, but satisfactory long-term results. Our report concurs with that of Ghika and Constatoyannis, that the Voa nucleus may well be an effective alternative when there is a damaged GPi. Our patient had presented with much worse preoperative condition, in comparison to those 2 reported cases, but had much earlier improvement, starting after 3 weeks and was able to stand up alone at 3 months and walked with assistance. The effects were stable throughout 18 months.

Nevertheless, since these are only sporadic cases and reflect the experience on just three patients, further studies are deemed necessary to achieve a more informed perception as to who would benefit from Voa DBS. The interest to the Voa has raised several studies on primary ${ }^{35-38}$ and secondary dystonia, ${ }^{39}$ where, we know the Gpi DBS is not as effective as in primary Dystonia.

\section{Conclusions}

Our patient with generalized postanoxic dystonia dramatically improved after Voa-DBS. This suggests, in continuity with the 2 cases from the literature, that Ventral oralis anterior nucleus of the thalamus is a potential target in Deep brain stimulation for the dystonia treatment, together with GPi, especially when the basal ganglia are selectively damaged, such as in postanoxic dystonia.

\section{Acknowledgments}

None.

\section{Conflicts of interest}

a. Maher Mansour has disclosed no conflicts of interest. 
b. Yasser-Mohamad Mansour has disclosed no conflicts of interest.

c. Leila Mansour has disclosed no conflicts of interest.

d. Janardan Vaidyanathan is an employee of Medtronic Middle East, Neuromodulation.

\section{References}

1. Coubes P, Roubertie A, Vayssierre N, et al. Early-onset dystonia: neurosurgical treatment with continuous bilateral stimulation of the internal globuspallidus. Neurology. 2000;54(suppl 3):A220.

2. Blond S, Caparros-Lefebvre D, Parker F, et al. Control of tremor and involuntary movement disorders by chronic stimulation of the ventral intermediate thalamic nucleus. J Neurosurg. 1992;77(1):62-68.

3. Bronte-Stewart H. Surgical therapy for dystonia. Curr Neurol Neurosci Rep. 2003;3(4):296-305.

4. Cooper IS. Twenty-year follow-up study of the neurosurgical treatment of dystonia musculorum deformans. Adv Neurol. 1976;14:423-452.

5. Derome PJ, Jedynak CP, Visot A, Delalande O. Treatment of abnormal movements by thalamic lesions. Rev Neurol. 1986;142(4):391-397.

6. Kupsch A, Benecke R, Muller J, et al. Pallidal deep-brain stimulation in primary generalized or segmental dystonia. $N$ Engl $\mathrm{J} \mathrm{Med}$. 2006;355(19):1978-1990.

7. Lang AE. Surgical treatment of dystonia. Adv Neurol. 1998;78:185-198.

8. Lozano AM, Kumar R, Gross RE, et al. Globus pallidus internus pallidotomy for generalized dystonia. Mov Disord. 1997;12(6):865-870

9. Mazars G, Merienne L, Cioloca C. Control of dyskinesias due to sensory deafferentation by means of thalamic stimulation. Acta Neurochir. 1980;30(suppl):239-243.

10. Ondo WG, Desaloms JM, Jankovic J, et al. Pallidotomy for generalized dystonia. Mov Disord. 1998;13(4):693-698.

11. Sellal F, Hirsch E, Barth P, et al. A case of symptomatic hemidystonia improved by ventroposterolateral pallidum electrostimulation. Mov Dis 1993;8(4):515-518.

12. Tasker RR, Doorly T, Yamashiro K. Thalamotomy in generalized dystonia Adv Neurol. 1988;50:615-631.

13. Vercueil L, Krack P, Pollak P. Results of deep brain stimulation for dystonia: a critical reappraisal. Mov Disord. 2002;17(Suppl 3):S89-S93.

14. Vidailhet M, Vercueil L, Houeto JL, et al. Bilateral pallidal deep brain stimulation in primary generalized dystonia: a prospective 3year follow-up study. Lancet Neurol. 2007;6(3):223-229.

15. Cury RG, Fraix V, Castrioto A, et al. Thalamic deep brain stimulation for tremor in Parkinson disease, essential tremor, and dystonia. Neurology. 2017;89(13):1416-1423.

16. Lenz FA, Jaeger CJ, Seike MS, et al. Thalamic single neuron activity in patients with dystonia: dystonia-related activity and somatic sensory reorganization. J Neurophysiol. 1999;82(5):2372- 2392.

17. Coubes P, Cif L, El Fertit H, et al. Electrical stimulation of the globus pallidus internus in patients with primary generalized dystonia: long-term results. J Neurosurg. 2004;101(2):189-194.

18. Krauss JK, Loher TJ, Weigel R, et al. Chronic stimulation of the globus pallidus internus for treatment of non-dYT1 generalized dystonia and choreoathetosis: 2- year follow up. J Neurosurg. 2003;98(4):785-792.

19. Loher TJ, Capelle HH, Kaelin-Lang A, et al. Deep brain stimulation for dystonia: outcome at long-term follow-up. J Neurol. 2008;255(6):881884
20. Tronnier VM, Fogel W. Pallidal stimulation for generalized dystonia Report of three cases. J Neurosurg. 2000;92(3):453-456.

21. Vidailhet M, Vercueil L, Houeto JL, et al. Bilateral deep-brain stimulation of the globus pallidus in primary generalized dystonia. $N$ Engl $\mathrm{J} \mathrm{Med}$. 2005;352:459-467.

22. Cif L, El Fertit H, Vayssiere N, et al. Treatment of dystonic syndromes by chronic electrical stimulation of the internal globus pallidus. J Neurosurg Sci. 2003;47(1):52-55.

23. Constantoyannis C, Kagadis G, Ellul J, Kefalopoulou Z, Chroni E. Nucleus Ventralis Oralis Deep Brain Stimulation in post anoxic Dystonia. Mov Dis. 2009;24(2):306-307.

24. Ghika J, Villemure JG, Miklossy J, et al. Postanoxic generalized dystonia improved by bilateral Voa thalamic deep brain stimulation. Neurology. 2002;58(2):311-313

25. Loher TJ, Hasdemir MG, Burgunder JM, et al. Long term follow-up study of chronic globus pallidus internus stimulation for posttraumatic hemidystonia. J Neurosurg. 2000;92(3):457-460.

26. Burke RE, Fahn S, Marsden CD, et al. Validity and reliability of a rating scale for the primary torsion dystonias. Neurology. 1985;35(1):73-77.

27. Guiot G, Arfel G, Derome P. Stereotaxic surgery of rest and attitude tremors. Gaz Med. 1968;75:4029-4056.

28. Schaltenbrand G, Wahren AE. Stereotaxic atlas of the human brain. New York: Thieme Verlag; 1982.

29. Hassler R. Anatomy of the thalamus. In: Schaltenbrand G, Baily P, editors Introduction to stereotaxis with an atlas of the human brain. Stuttgart: Thieme; 1959. P. 230-290.

30. Ilinsky I, Kultas-Ilinsky K. Motor Thalamic Circuits in Primates with Emphasis on the Area Targeted in Treatment of Movement Disorders. Mov Disord. 2002;17(Suppl 3):S9-S14.

31. Kostic VS, Stojanovic-Svetel M, Kacar A. Symptomatic dystonias associated with structural brain lesions: report of 16 cases. Can J Neurol Sci. 1996;23(1):53-56.

32. Benabid AL, Benazzouz A, Hoffmann D, et al. Long-term electrical inhibition of deep brain targets in movement disorders. Mov Disord. 1998;3:119-125.

33. Vercueil L, Pollak P, Fraix V, et al. Deep brain stimulation in the treatment of severe dystonia. J Neurol. 2001;248(8):695-700.

34. Tasker RR, Organ LW, Hawrylyshyn PA. The thalamus and midbrain of man. A physiological atlas using electrical stimulation. The Journal of Nervous and Mental Disease. 1983;171(11):694-695.

35. Pauls KA, Hammesfahr S, Moro E, et al. Deep brain stimulation in the ventrolateral thalamus/subthalamic area in dystonia with head tremor. Mov Disord. 2014;29(7):953-959.

36. Buhmann C, Moll CK, Zittel S, et al. Deep brain stimulation of the ventrolateral thalamic base and posterior subthalamic area in dystonic head tremor. Acta Neurochir Suppl. 2013;117:67-72.

37. Mure H, Morigaki R, Koizumi H, et al. Deep brain stimulation of the thalamic ventral lateral anterior nucleus for DYT6 dystonia. Stereotact Funct Neurosurg. 2014;92(6):393-396.

38. Slotty PJ, Poologaindran A, Honey CR. A prospective, randomized, blinded assessment of multitarget thalamic and pallidal deep brain stimulation in a case of hemidystonia. Clin Neurol Neurosurg. 2015;138:16-19.

39. Thalamic Deep Brain Stimulation for Secondary Dystonia in Children and Young Adults (DBSVop). USA: University of California, San Francisco, ClinicalTrials.gov. 2019. 